PROCEEDINGS OF THE

AMERICAN MATHEMATICAL SOCIETY

Volume 127, Number 9, Pages 2679-2683

S 0002-9939(99)04899-6

Article electronically published on April 15, 1999

\title{
ON THE PRODUCT OF TWO GENERALIZED DERIVATIONS
}

\author{
MOHAMED BARRAA AND STEEN PEDERSEN
}

(Communicated by Palle E. T. Jorgensen)

\begin{abstract}
Two elements $A$ and $B$ in a ring $\mathfrak{R}$ determine a generalized derivation $\delta_{A, B}$ on $\mathfrak{R}$ by setting $\delta_{A, B}(X)=A X-X A$ for any $X$ in $\mathfrak{R}$. We characterize when the product $\delta_{C, D} \delta_{A, B}$ is a generalized derivation in the cases when the ring $\mathfrak{R}$ is the algebra of all bounded operators on a Banach space $\mathcal{E}$, and when $\mathfrak{R}$ is a $C^{*}$-algebra $\mathfrak{A}$. We use these characterizations to compute the commutant of the range of $\delta_{A, B}$.
\end{abstract}

\section{IntRoduCtion}

Let $\mathfrak{B}$ be a ring or an algebra. A derivation on $\mathfrak{B}$ is an additive (linear) map $\delta: \mathfrak{B} \rightarrow \mathfrak{B}$ satisfying $\delta(X Y)=\delta(X) Y+X \delta(Y)$ for all $X$ and $Y$ in $\mathfrak{B}$. Consequences of assuming

(*) the product $\delta_{1} \delta_{2}$ of two derivations $\delta_{1}$ and $\delta_{2}$ again is a derivation

have been investigated in a number of papers. Posner $[\mathrm{Po}]$ proved that if $\mathfrak{B}$ is a prime ring of characteristic not 2, then $(*)$ implies $\delta_{1}=0$ or $\delta_{2}=0$. This result has been reproved in other papers, e.g., [FS] and [Wi], under the stronger assumption that $\mathfrak{B}=\mathcal{L}(\mathcal{H})$, the algebra of all bounded linear operators on the Hilbert space $\mathcal{H}$. If $\mathfrak{B}=\mathfrak{A}$ is a $C^{*}$-algebra, then $(*)$ implies $\delta_{1} \delta_{2}=0$ [Ma1] (a result of this type is proved in [Ped] for unbounded densely defined derivations), and $(*)$ together with $\delta_{1}=\delta_{2}=\delta$ implies $\delta=0$. In [Wi] the range of a derivation on $\mathcal{L}(\mathcal{H})$ is investigated, a major tool in [Wi] is the result mentioned above. It is known (e.g., [Ch], [Ka], [Sa1], [Se]) that a derivation of $\mathcal{L}(\mathcal{E})$ is inner, i.e., there exists an element $A$ in $\mathcal{L}(\mathcal{E})$, the algebra of all bounded linear operators on the Banach space $\mathcal{E}$, so that $\delta(X)=\delta_{A}(X)=A X-X A$ for all $X$ in $\mathcal{L}(\mathcal{E})$. It is also known (a result of Sakai) [Pe], [Sa2] that, if $\delta$ is a derivation of a $C^{*}$-algebra $\mathfrak{A}$ then, $\delta=\delta_{A}$ for some $A$ in the enveloping von Neumann algebra of $\mathfrak{A}$.

For two operators $A$ and $B$ in $\mathcal{L}(\mathcal{E})$ (or in the multiplier algebra $M(\mathfrak{A})$ of $\mathfrak{A}$ ), we say that the operator $\delta_{A, B}(X)=A X-X B$ is a generalized derivation. It is shown in $[\mathrm{Br}]$ that, if the product of two derivations of an prime ring of characteristic not 2 is a generalized derivation, then one of the derivations must be zero. We characterize when the product $\delta_{C} \delta_{A, B}$ of a derivation and a generalized derivation

Received by the editors December 30, 1996 and, in revised form, November 20, 1997.

1991 Mathematics Subject Classification. Primary 47B47, 46L40.

Key words and phrases. Derivation, generalized derivation, elementary operator, $C^{*}$-algebra.

(C)1999 American Mathematical Society 
on $\mathcal{L}(\mathcal{E})$ is a derivation. In contrast to the product of two derivations, the product $\delta_{C} \delta_{A, B}$ can be a non-zero derivation. As a consequence of the characterization we obtain information about the commutant of the range of a generalized derivation. We also apply the results for $\mathfrak{B}=\mathcal{L}(\mathcal{E})$ to obtain a characterization for the case when $\mathfrak{B}=\mathfrak{A}$ is a $C^{*}$-algebra. The later characterization is strong enough to yield the results, mentioned above, characterizing when the products $\delta \delta$ and $\delta_{1} \delta_{2}$ are derivations on a $C^{*}$-algebra as easy corollaries. In the final section we consider the product of two generalized derivations.

We refer the reader to [Pe] for background information about $C^{*}$-algebras, and to $[\mathrm{Pe}]$ and [Sa2] for the theory of derivations in operator algebras. Generalized derivations have been studies extensively, see e.g., [CF], [FS], and [Ma2].

\section{The COMmutant OF THE RANGE}

In this part of the paper we consider bounded linear operators on a Banach space $\mathcal{E}$, we show that there exist $C$ in $\mathcal{L}(\mathcal{E}) \backslash \mathbb{C} I$ such that $\delta_{C} \delta_{A, B}$ is a derivation, if and only if $A+B \in \mathbb{C} I$. We use this to compute the commutant, in $\mathcal{L}(\mathcal{E})$, of the range of $\delta_{A, B}$. The first result is an immediate consequence of Theorem 5 below.

Theorem 1. Let $\mathcal{E}$ be a Banach space and let $A$ and $B$ be in $\mathcal{L}(\mathcal{E})$. There exist $C$ in $\mathcal{L}(\mathcal{E}) \backslash \mathbb{C} I$ so that $\delta_{C} \delta_{A, B}$ is a derivation if and only if $A+B \in \mathbb{C} I$. More precisely, when $A+B \in \mathbb{C} I$, the possible choices for $C$ are given by

1. if $A-B \in \mathbb{C} I$, then $C$ can be any element of $\mathcal{L}(\mathcal{E}) \backslash \mathbb{C} I$

2. if $A-B \notin \mathbb{C} I$, then $C=a(A-B)+b I$, where $a, b \in \mathbb{C}$ and $a \neq 0$.

Remark. Theorem 1 remains true for standard algebras. A standard algebra is a subalgebra of $\mathcal{L}(\mathcal{E})$ containing all the finite rank operators.

Corollary 2. If $A$ and $B$ are in $\mathcal{L}(\mathcal{E})$, then the commutant in $\mathcal{L}(\mathcal{E})$ of the range of $\delta_{A, B}$ is:

1. $\mathbb{C} I$, if $A+B \notin \mathbb{C} I$ or if $A+B \in \mathbb{C} I$ and either $A-B \in \mathbb{C} I \backslash\{0\}$ or $(A-B)^{2} \notin \mathbb{C} I$

2. $\mathcal{L}(\mathcal{E})$, if $A+B \in \mathbb{C} I$ and $A-B=0$;

3. $\{a(A-B)+b I: a, b \in \mathbb{C}\}$, if $A+B \in \mathbb{C} I, A-B \notin \mathbb{C} I$ and $(A-B)^{2} \in \mathbb{C} I$.

Proof. First observe that an element $C$ of $\mathcal{L}(\mathcal{E})$ is in the commutant $\left(\operatorname{Ran} \delta_{A, B}\right)^{\prime}$ if and only if $\delta_{C} \delta_{A, B}=0$. Hence, if $C$ is in the commutant of the range of $\delta_{A, B}$ then $\delta_{C} \delta_{A, B}$ is a derivation. The proof of the corollary is divided into three cases.

1. $A+B \notin \mathbb{C} I$. In this case Theorem 1 implies any $C$ in the commutant of the range of $\delta_{A, B}$ is in $\mathbb{C} I$. Clearly, $\mathbb{C} I$ is a subset of the commutant.

2. $A+B \in \mathbb{C} I$ and $A-B \in \mathbb{C} I$. In this case $A=a I$ and $B=b I$, hence $\delta_{A, B}(X)=(a-b) X$ for all $X$ in $\mathcal{L}(\mathcal{E})$, the remaining details are left for the reader.

3. $A+B \in \mathbb{C} I$ and $A-B \notin \mathbb{C} I$. If follows from Theorem 1 that

$$
\left(\operatorname{Ran} \delta_{A, B}\right)^{\prime} \subseteq\{a(A-B)+b I: a, b \in \mathbb{C}\}
$$


It follows from (4), (5) and (7) below that $A=a C+b I$ and $B=-a C+c I$ for some scalars $a, b$, and $c$, hence we have

$$
\begin{aligned}
\left(\text { Ran } \delta_{A, B}\right)^{\prime} & \ni C \text { iff } \\
\delta_{C} \delta_{A, B}=\delta_{a C^{2}+(b-c) C} & =0 \text { iff } \\
a C^{2}+(b-c) C & \in \mathbb{C} I \text { iff } \\
(2 a C)^{2}+2(b-c) \cdot 2 a C & \in \mathbb{C} I \text { iff } \\
(A-B-(b-c) I)^{2}+2(b-c)(A-B-(b-c) I & \in \mathbb{C} I \text { iff } \\
(A-B)^{2} & \in \mathbb{C} I .
\end{aligned}
$$

Again a few easy details are left for the reader.

\section{Applichtions to $C^{*}$-Algebras}

Let $\mathfrak{A}$ be a $C^{*}$-algebra, and let $A$ and $B$ be in the multiplier algebra $M(\mathfrak{A})$ of $\mathfrak{A}$. Let $\pi$ be the reduced atomic representation of $\mathfrak{A}$, that is, $\pi=\sum_{t \in \hat{A}}^{\otimes} \pi_{t}$ on the Hilbert space $\mathcal{H}=\sum_{t \in \hat{A}}^{\otimes} \mathcal{H}_{t}$. It is well known that $\pi$ is faithful, and that the double commutant, $\pi(\mathfrak{A})^{\prime \prime}$, of $\pi(\mathfrak{A})$ satisfies

$$
\pi(\mathfrak{A})^{\prime \prime}=\prod_{t \in \hat{A}} \mathcal{L}\left(\mathcal{H}_{t}\right) .
$$

See, for example, $[\mathrm{Pe}]$ for more details about the reduced atomic representation. Let $C$ in the enveloping von Neumann algebra of $\mathfrak{A}$ be so that $\delta_{C} \delta_{A, B}$ is a derivation of $\mathfrak{A}$, and pick $D$ in the enveloping von Neumann algebra, so that $\delta_{C} \delta_{A, B}=\delta_{D}$. Then for $X$ in $\mathfrak{A}$ we have

$$
C(A X-X B)-(A X-X B) C=D X-X D .
$$

For $t \in \hat{A}$ we can apply $\pi_{t}$ to (1) and get

$$
\begin{aligned}
& \pi_{t}(C)\left(\pi_{t}(A) \pi_{t}(X)-\pi_{t}(X) \pi_{t}(B)\right) \\
& \quad-\left(\pi_{t}(A) \pi_{t}(X)-\pi_{t}(X) \pi_{t}(B)\right) \pi_{t}(C) \\
& =\pi_{t}(D) \pi_{t}(X)-\pi_{t}(X) \pi_{t}(D) .
\end{aligned}
$$

If $\pi_{t}(C)$ is not a scalar multiple of the identity on $\mathcal{H}_{t}$, then we can apply Theorem 1 to the operators $\delta_{\pi_{t}(C)}$ and $\delta_{\pi_{t}(A), \pi_{t}(B)}$ to conclude that $\pi_{t}(A+B)=\pi_{t}(A)+\pi_{t}(B)$ is a scalar times the identity on $\mathcal{H}_{t}$. It follows from the faithfulness of the reduced atomic representation that $A+B$ is a scalar times the identity in $M(\mathfrak{A})$. It is clear that if $\pi_{t}(C)$ is a scalar multiple of the identity on $\mathcal{H}_{t}$, then $\pi_{t}(A)$ and $\pi_{t}(B)$ can be arbitrary operators on $\mathcal{H}_{t}$. We have shown:

Corollary 3. If $A, B$ and $C$ are operators in the multiplier algebra $M(\mathfrak{A})$ of some $C^{*}$-algebra $\mathfrak{A}$, then $\delta_{C} \delta_{A, B}$ is a derivation on $\mathfrak{A}$ if and only if, for each $t \in \hat{A}$, either $\pi_{t}(A+B)$ or $\pi_{t}(C)$ is a scalar multiple of the identity on $\mathcal{H}_{t}$.

Immediate consequences of Corollary 3 are the known results that if $\delta$ is a derivation on a $C^{*}$-algebra so that $\delta \delta$ also is a derivation, then $\delta=0$; and if $\delta_{1}, \delta_{2}$, and $\delta_{1} \delta_{2}$ all are derivations on a $C^{*}$-algebra, then $\delta_{1} \delta_{2}=0$. 
Corollary 4. Let $\mathfrak{A}$ be a $C^{*}$-algebra and let $A$ and $B$ be in the multiplier algebra $M(\mathfrak{A})$ of $\mathfrak{A}$. Then $\left(\operatorname{Ran} \delta_{A, B}\right)^{\prime} \neq \mathbb{C} I$ if and only if there is an irreducible representation $\pi$ of $\mathfrak{A}$ so that either $\pi(A)=\pi(B) \in \mathbb{C} I$ or $\pi(A+B) \in \mathbb{C} I, \pi(A-B) \notin \mathbb{C} I$ and $\pi(A-B)^{2} \in \mathbb{C} I$.

Proof. The proof is based on Corollary 2 instead of Theorem 1, otherwise it is similar to the proof of Corollary 3.

When defining $\delta_{A, B}$ on a $C^{*}$-algebra $\mathfrak{A}$ is it not really necessary that $A$ and $B$ are in the multiplier algebra $M(\mathfrak{A})$. We only need that $A$ and $B$ are in the enveloping von Neumann algebra and that $\delta_{A, B}$ maps $\mathfrak{A}$ into itself.

\section{The product on $\mathcal{L}(\mathcal{E})$}

In this section we investigate when the product of two generalized derivations again is a generalized derivation. In particular we prove the following generalization of Theorem 1.

Theorem 5. Let $A, B, C$, and $D$ be bounded operators on a Banach space $\mathcal{E}$.

1. If $A \notin \mathbb{C} I$ and $B \notin \mathbb{C} I$, then $\delta_{C, D} \delta_{A, B}$ is a generalized derivation if and only if $C=a A+c I$ and $D=-a B+d I$ for some scalars $a, c$, and $d$;

2. If $A \in \mathbb{C} I$ and $B \notin \mathbb{C} I$, then $\delta_{C, D} \delta_{A, B}$ is a generalized derivation if and only if $C \in \mathbb{C} I$

3. If $A \notin \mathbb{C} I$ and $B \in \mathbb{C} I$, then $\delta_{C, D} \delta_{A, B}$ is a generalized derivation if and only if $D \in \mathbb{C} I$;

4. If $A \in \mathbb{C} I$ and $B \in \mathbb{C} I$, then $\delta_{C, D} \delta_{A, B}$ is a generalized derivation.

Proof. We will only prove part 1 , the proofs of parts 2 and 3 are similar, but simpler, and part 4 is trivial. Suppose $A, B \notin \mathbb{C} I$ and $\delta_{C, D} \delta_{A, B}=\delta_{E, F}$. Let $G=C A-E$ and $H=B D-F$; then

$$
C X B y+A X D y=G X y-X H y
$$

for all $X \in \mathcal{L}(\mathcal{E})$. Consider the element $X=x \otimes f$ of $\mathcal{L}(\mathcal{E})$ determined by $X y=$ $f(y) x$. Then (2) becomes

$$
f(B y) C x+f(D y) A x=f(y) G x-f(H y) x
$$

for all $x$ and $y$ in $\mathcal{E}$ and all $f$ in $\mathcal{E}^{\prime}$. Since $B \notin \mathbb{C} I$ we can find $y$ in $\mathcal{E}$ so that $y$ and $B y$ are linearly independent, hence we can pick $f$ in $\mathcal{E}^{\prime}$ so that $f(y)=0$ and $f(B y)=-1$. Using these choices in (3) we see that $C x=f(D y) A x+f(H y) x$ for all $x$ in $\mathcal{E}$. It follows that

$$
C=a A+c I
$$

for some scalars $a$ and $c$. Considering the adjoint of (2) it follows similarly that

$$
D=b B+d I
$$

for some scalars $b$ and $d$. It remains to show that $a+b=0$. Let $K=G-d A$ and $L=H+c B$. Substituting (4) and (5) into (2) we get

$$
(a+b) A X B y=K X y-X L y
$$

for all $y$ in $\mathcal{E}$ and all $X \in \mathcal{L}(\mathcal{E})$. If $y$ and $f$ are so that $f(y)=1$ and $f(B y)=0$, then letting $X=x \otimes f$ in (6) we get $K \in \mathbb{C} I$. Similarly, considering adjoints, we 
get $L \in \mathbb{C} I$. Hence, if $X=x \otimes f$ and $f(y)=0$, and $f(B y)=1$ then it follows from (6) that $(a+b) A \in \mathbb{C} I$. Since, $A \notin \mathbb{C} I$ we conclude that

$$
a+b=0 .
$$

The converse is trivial.

Remark. It is easy to obtain a variety of special cases of Theorem 5 by asking when $\delta_{C, D} \delta_{A, B}=\delta_{E, F}$ and one or more of the following hold: $A=B, C=D$, or $E=F$. For example

1. if $\delta_{C, C} \delta_{A, A}=\delta_{E, F}$, then $C \in \mathbb{C} I$ or $A \in \mathbb{C} I$ and $E=F \in \mathbb{C} I$ (this generalization of Posner's theorem was also observed in [Br]);

2. if $\delta_{C, C} \delta_{A, B}=\delta_{E, F}$, then $A+B \in \mathbb{C} I$ and $E=F$ (this is a generalization of Theorem 1 above);

3. if $\delta_{C, D} \delta_{A, A}=\delta_{E, F}$, then $C+D \in \mathbb{C} I$ and $E=F$.

As in section 3 one can extend the results in this section to obtain a characterization of when the product of two generalized derivations of a $C^{*}$-algebra is a generalized derivation.

\section{REFERENCES}

[Br] M. Brešar, On the distance of the composition of two derivations to the generalized derivations, Glasgow Math. J. 33 (1991), pp. 89-93. MR 92b:46071

[Ch] P. R. Chernoff, Representations, automorphisms, and derivations of some operator algebras, J. Funct. Anal. 12 (1972), pp. 275-289. MR 50:2934

$[\mathrm{CF}] \quad$ R. E. Curto and L. A. Fialkow, The spectral picture of $\left(L_{A}, R_{B}\right)$, J. Funct. Anal. 71 (1987), pp. 371-392. MR 88c:47006

[FS] C. K. Fong and A. R. Sourour, On the operator identity $\sum A_{k} X B_{k} \equiv 0$, Canad. J. Math. 31 (1979), pp. 845-857. MR 80h:47020

[Ka] R. V. Kadison, Derivations on operator algebras, Ann. of. Math. 83 (1966), pp. 280-293. MR 33:1747

[Ma1] M. Mathieu, Properties of the product of two derivations, Canad. Math. Bull. 32 (1989), pp. 490-497. MR 90k:46140

[Ma2] M. Mathieu, Elementary operators on prime $C^{*}$-algebras, I, Math. Ann. 284 (1989), pp. 223-244. MR 90h:46092

[Pe] G. K. Pedersen, $C^{*}$-algebras and their automorphism groups, Academic Press, London, 1979.

[Ped] S. Pedersen, The product of two unbounded derivations, Canad. Math. Bull. 33 (1990), pp. 345-348. MR 92a:46081

[Po] E. C. Posner, Derivations in prime rings, Proc. Amer. Math. Soc. 8 (1957), pp. 1093-1100. MR 20:2361

[Sa1] S. Sakai, Derivations on $W^{*}$-algebras, Ann. of. Math. 83 (1966), pp. 273-279. MR 33:1748

[Sa2] S. Sakai, Operator algebras in dynamical systems, Encyclopedia of Mathematics and its Applications, vol. 41, Cambridge University Press, Cambridge, 1991. MR 92h:46099

[Se] P. Semrl, Ring derivations on standard operator algebras, J. Funct. Anal. 112 (1993), pp. 318-324. MR 94h:47084

[Wi] J. P. Williams, On the range of a derivation, Pacific J. Math. 38 (1971), pp. 273-279. MR 46:7923

Departement de Mathematiques, Faculte des Sciences-Semlalia, University Cadi AyYad, B.P.: S. 15, 40000 Marrakech, Marocco

Department of Mathematics, Wright State University, Dayton, Ohio 45435

E-mail address: steen@math.wright.edu 